days of withdrawing TNF signals. Dose-response studies indicated that TNF-treated T cells required TCR engagement with higher peptide concentrations for longer periods of time for commitment to IL-2 production. Subsequent experiments revealed that chronic TNF exposure led to a reversible loss of TCR $\zeta$ chain expression, in part through a reduction in gene transcription. Downregulation of TCR $\zeta$ impaired TCR/CD3 assembly and expression at the cell surface, and uncoupled membrane proximal tyrosine phosphorylation events, including phosphorylation of the TCR $\zeta$ chain itself, CD $3 \epsilon$, ZAP-70 protein tyrosine kinase and LAT. Intracellular $\mathrm{Ca}^{2+}$ mobilisation was also suppressed in TNF-treated T cells. Signals transduced through a single chain $\mathrm{Fv}$ antibody/FcR $\gamma$ chain chimeric receptor that recognises native type II collagen, and which utilises TCR $\zeta$ for IL-2 gene transcription, were also attenuated. We propose that TNF may contribute to T cell hyporesponsiveness in chronic inflammatory and infectious diseases, such as rheumatoid arthritis, by mechanisms which include downregulation of TCR $\zeta$ expression. We speculate that by uncoupling proximal TCR signals TNF could also interrupt mechanisms of peripheral tolerance which are dependent upon intact TCR signal transduction pathways.

Objectives

Methods

Results

Conclusion

\section{OP0032 INVASION OF SYNOVIOCYTES IS INHIBITED BY GENE TRANSFER OF TNF-BP OR IL10 IN AN IN VITRO INVASION MODEL}

${ }^{1}$ EJ Pieterman, ${ }^{1} \mathrm{PH}$ Goossens, ${ }^{2} \mathrm{WH}$ vd Laan, ${ }^{1} \mathrm{AL}$ Huidekoper, ${ }^{3} \mathrm{MJ}$ Rabelink, ${ }^{3} \mathrm{RC}$ Hoeben, ${ }^{1} \mathrm{FC}$ Breedveld, 'TW Huizinga. 'Rheumatology: 'aubius Laboratory, TNO Prevention and Health, Leiden, The Netherlands; ${ }^{3}$ Department of Molecular Cell Biology, Leiden University Medical Centre

\subsection{6/annrheumdis-2001.817}

Background In RA fibroblast like synoviocytes (FLS) degrade and invade into adjacent cartilage. An in vivo model is the SCID-mouse/Human cartilage/synoviocyt model (S. Gay). Previous studies indicate that the invasive behaviour of fibroblast like synoviocytes can be tested in vitro in a matrigel transwell system. Matrigel, mainly composed of laminin and collagen IV, serves as a model for cartilage.

Objectives The aim of this study was to compare the invasive behaviour of FLS from RA and osteoarthritis (OA) patients and to investigate the effect of adenoviral (Ad) transfer of genes encoding IL-10 and TNF-binding protein (p55) (TNF-BP) on the invasive behaviour of FLS from RA patients.

Methods FLS from 43 RA and 28 OA patients obtained from synovial tissue harvested at joint replacement surgery, were seeded at confluency in serum free medium on top of a matrigel coated transwell filter with $8 \mu \mathrm{m}$ pores. Medium with 10\% Fetal Calf Serum and 10\% Human Serum was used in the lower compartment. Three days post incubation cells were fixated, stained and the invaded synoviocytes on the lower side of the filter were counted. To test the effect of IL10 and TNF-BP, RA synoviocytes were infected overnight with $5,10,50$ and 100 plaque forming units (pfu)/cel of Ad. IL10, Ad. TNF-BP or Ad. luciferase (negative control) and then tested in the invasion model.

Results Significantly more RA synoviocytes invaded through the matrigel (median $=4035$ cells) as compared to OA synoviocytes (median $=1900$ cells; $\mathrm{p}<0.001)$. IL10 and TNF-BP gene transfer both resulted in a dose dependent inhibition of invasion with a maximal inhibition of $93.7 \%( \pm 10.7)$ and $86.6 \%( \pm$ $14.5)$ respectively, while luciferase gene transfer showed a maximal inhibition of $17.6 \%$ ( \pm 1.7$)$.

Conclusion In conclusion, the invasive behaviour of FLS can be studied in the matrigel transwell system. This assay discriminates between the invasive behaviour of RA and OA FLS. The invasive behaviour of RA synoviocytes can be strongly inhibited by IL10 indicating that IL10 is able to downregulate the proteins involved in invasive growth. The inhibitive effect of TNF-BP indicates that continuous production of TNF is involved in invasive behaviour of synoviocytes.

\section{OP0033 SYSTEMIC ADENOVIRUS MEDIATED GENE THERAPY USING A CHIMERIC SOLUBLE IL-1 RECEPTOR TYPE II ? IGG PROTEIN REDUCES ACUTE INFLAMMATION IN ANTIGEN-INDUCED ARTHRITIS}

J Dudler, R Salvi, N Busso, V Chobaz-Péclat, A So. Service de Rhumatologie, CHUV, Lausanne, Switzerland

10.1136/annrheumdis-2001.818

Background The anti-inflammatory effects of IL-1 inhibition are well established but most studies have focused on the endogenous IL-1 receptor antagonist (IL-1Ra) protein. However, IL1ra has a very short plasma half-life and hundred-fold excess over IL-1 is required to obtain a $50 \%$ inhibition of IL-1 responses. Another naturally occurring inhibitor, soluble IL-1 receptor type II (sIL-1R2) may offer a higher IL-1 neutralising activity and several studies have emphasised its potential function as a regulatory "decoy" for IL1b.

Objectives To construct soluble IL-1 receptor type II chimeric protein with an IgG heavy chain and to evaluate its therapeutic potential in a murine arthritis model using a gene therapy approach.

Methods The murine IL-1R2 extracellular domain was cloned using a RT-PCR protocol from total cytoplasmic RNA, sequenced and a chimeric expressing gene constructed by the addition of a XbaI fragment encoding the constant and hinge regions of a murine IgG1 heavy chain. The IL-1R2IgG coding insert was subcloned into an E1-deleted adenoviral shuttle expression plasmid under the control of a CMV promoter and the resulting plasmid used to construct a standard first generation E1-deleted recombinant adenovirus (Ad-sIL1R2 Ig) using standard homologous recombination techniques in 293 cells. Stock viral preparations were produced in 293 cells and purified by ultracentrifugation in two $\mathrm{CsCl}^{2}$ gradients. Viral titer $(\mathrm{pfu} / \mathrm{ml})$ was determined by plaque-assay. An EGFP (enhanced green fluorescent protein) expressing recombinant adenovirus (Ad-EGFP) was prepared using a similar protocol. Expression was confirmed by Western blotting using transient expression on HeLa cells under serum free-conditions.

The therapeutic effects of this approach was tested in the acute murine antigen-induced arthritis model (AIA).

Results Western blotting under reducing and nonreducing conditions confirmed expression and dimerization of the chimeric protein. More importantly, adenovirus-mediated systemic expression of sIL1R2 Ig suppressed acute inflammation in vivo in murine AIA. Mice were injected into the tail vein with 109 pfu AdsIL1R2Ig or Ad-EGFP three days before induction of arthritis by intraarticular injection of $100 \mu \mathrm{g}$ mBSA into the right knee. The left knee was injected with PBS as a control. Joint inflammation 